\title{
Extinction and retraining of simultaneous and successive flavor conditioning
}

\author{
THOMAS HIGGINS and ROBERT A. RESCORLA \\ University of Pennsylvania, Philadelphia, Pennsylvania
}

\begin{abstract}
In three experiments, rats received pairings of flavor conditioned stimuli with polycose unconditioned stimuli, in either a simultaneous or a sequential relation. Both temporal relations produced excellent conditioned increases in consumption of the flavors. Separate presentation of the flavors resulted in extinction in both cases. However, restoring the pairing of the flavor with polycose resulted in reconditioning only with the sequential, not with the simultaneous, relation.
\end{abstract}

The joint presentation of two stimuli, A and B, commonly results in the formation of an association between them. Moreover, despite wide variation in the nature of $\mathrm{A}$ and $\mathrm{B}$, and in the details of the circumstances in which they are presented, the associations developed share many basic features. For instance, within the domain of Pavlovian conditioning, in which $\mathrm{A}$ is a relatively neutral stimulus and $\mathrm{B}$ is a more powerful stimulus, it is virtually universal to observe that the pairing of $\mathrm{A}$ and $\mathrm{B}$ results in the acquisition of responding to A. Similarly, subsequent separate presentation of A without B produces deterioration of that responding, extinction. Moreover, pairing A and B once again yields reestablishment of responding to A. Indeed, that retraining is frequently more rapid than initial training. These observations of acquisition, extinction, and retraining are so universal that they are considered by textbook writers to be inevitable consequences of pairing A and B (e.g., Domjan, 2003; Schwartz, Wasserman, \& Robbins, 2002).

In light of this general pattern, it is surprising to find a report of a failure to observe retraining in a preparation that otherwise produces rapid acquisition and extinction. In a series of investigations of sensory preconditioning, in which A and B were both relatively neutral, Rescorla (1981) and Rescorla and Durlach (1981) described evidence that simultaneous presentations of two flavor stimuli produced especially strong associations between A and B. Moreover, those associations showed acquisition and extinction functions like those normally observed, when A and B were presented together or A was presented separately (Rescorla \& Freberg, 1978; Westbrook et al., 1995). However, Rescorla (1981) also reported the surprising results of a retraining experiment by Freberg (1979) in which she found no evidence that such simul-

This research was supported by National Institute of Health Grant R01 MH 67848. The authors are grateful for discussions with Dan Gottlieb. Correspondence concerning this article should be addressed to R. A. Rescorla, Department of Psychology, University of Pennsylvania, 3720 Walnut Street, Philadelphia, PA, 19104 (e-mail: rescorla@cattell.psych.upenn.edu). taneous associations could be retrained if $\mathrm{A}$ and $\mathrm{B}$ were once again presented together after extinction.

The reasons for this deviant finding are not clear. Rescorla (1981) speculated that this result might mean that simultaneous presentation generates a different kind of association, following different rules. However, he provided no direct comparisons with the more conventional sequential presentation of stimuli. Indeed, he reported relatively little associative learning at all with sequential presentation of stimuli in this situation. Alternatively, these results might have been due to Freberg's (1979) use of relatively neutral A and B stimuli, which then required the subsequent induction of value in $\mathrm{B}$ in order to detect the performance to $\mathrm{A}$ indicative of an $\mathrm{A}-\mathrm{B}$ association. It is quite possible that it is the relative neutrality of $A$ and $\mathrm{B}$, and the fragility of sensory preconditioning, that prevented retraining.

The goal of the present series of experiments was to replicate and to explore further Freberg's failure to find retraining. These experiments employed a more conventional Pavlovian procedure in which one stimulus was originally neutral and another more powerful, to explore retraining under simultaneous and sequential presentation. For this purpose, a conditioning procedure was selected that could be expected to result in substantial learning under both temporal relations. Rats received pairings of flavor stimuli with a positively valued substance, polycose. Preliminary work in this laboratory has confirmed the report that gustatory stimuli can be similarly conditioned with both simultaneous and sequential presentation of polycose (e.g., Capaldi, Hunter, \& Lyn, 1997). In both cases, a flavor previously paired with polycose shows enhanced consumption.

In Experiment 1, acquisition, extinction, and reacquisition were conducted with simultaneous presentation of a flavor and the delivery of polycose. The intention was to determine whether Freberg's finding of failure to retrain could be replicated with a more standard Pavlovian procedure in which one stimulus is relatively neutral and the other relatively strong. In Experiment 2, similar acquisition, extinction, and reacquisition were conducted 
with sequential presentation of the same stimuli. The intention was to confirm that sequential presentation would yield the conventional ability to retrain with this conditioning preparation. In Experiment 3, a direct withinsubjects comparison was made between the sensitivity of sequential and the sensitivity of simultaneous associations to retraining.

\section{EXPERIMENT 1}

This experiment examined acquisition, extinction, and retraining procedures using a flavor as a conditioned stimulus (CS) and concentrated polycose as the simultaneous unconditioned stimulus (US). Hungry rats received presentations of an almond flavor mixed in a concentrated $20 \%$ polycose solution. Then one group of animals received repeated nonreinforced presentations of the almond flavor, mixed in water. That in turn was followed by retraining and testing. Comparison groups received either training but no extinction or training and extinction but no retraining. A control group received unpaired presentations of almond and $20 \%$ polycose.

\section{Method}

\section{Subjects and Apparatus}

The subjects were 32 male Sprague-Dawley rats, about 150 days old. They had previously participated in a magazine-approach experiment using auditory and visual stimuli and a food pellet reinforcer, during which they were maintained on an $80 \%$ food deprivation schedule. They were returned to ad-lib feeding for 2 weeks prior to the present experiment and then redeprived to $80 \%$ of their new weights at the end of that ad-lib period. Ad-lib access to water was provided except during trials. All experimental procedures took place in individual home cages (Wahmann LC385/SA). Affixed to the outside of each cage was a holder permitting the mounting of a 50 -ml centrifuge tube with a rubber stopper and stainless steel drinking spouts. All experimental stimuli were delivered in those tubes. The US consisted of $5 \mathrm{~min}$ of exposure to $45 \mathrm{ml}$ of distilled water containing a $20 \%$ polycose (Albott Laboratories, Columbus, $\mathrm{OH}$ ) solution. The CS consisted of $1 \%$ almond (McCormick pure almond extract) solution. This was presented mixed in $20 \%$ polycose solution (acquisition), in distilled water (extinction), or in a $2 \%$ polycose solution (unpaired control group).

\section{Procedure}

On each experimental day, the water bottle was removed from each cage $30 \mathrm{~min}$ prior to the trial and then replaced $30 \mathrm{~min}$ after the trial. With the return of the water, each animal was weighed and given its daily food ration.

Preexposure. On the first experimental day, all animals were given preexposure to the US. They received $20 \mathrm{~min}$ of access to $45 \mathrm{ml}$ of a $20 \%$ polycose solution. The intention was to familiarize them with the US so as to guarantee its ready consumption during conditioning.

Conditioning. Over the next 18 days, the animals in Groups Extinction $(n=8)$, Retrain $(n=8)$, and No Extinction $(n=8)$ received a Pavlovian conditioning procedure in which an almond solution was paired with polycose. On each conditioning day, the animals received 5 min of access to a mixture of $1 \%$ almond with $20 \%$ polycose. Conditioning trials were conducted on 9 of the 18 days, with simple exposure to $5 \mathrm{~min}$ of distilled water on the other 9 days. This was done to accommodate the demands of the procedure in an unpaired control group.

The 8 animals in Group Unpaired received presentations of almond and the $20 \%$ polycose on separate days. On days when the ex- perimental animals received paired almond-polycose presentation, the unpaired animals received $5 \mathrm{~min}$ of exposure to the $1 \%$ almond solution mixed with $2 \%$ polycose. Almond was mixed with this weaker solution to guarantee that it would be consumed in amounts similar to that in the experimental groups. The weaker polycose solution has been found in preliminary work to encourage consumption without yielding substantial conditioning. On the days when the experimental animals received exposure to water, the unpaired animals received $5 \mathrm{~min}$ of access to a $20 \%$ polycose solution. The treatments were administered in a double alternation pattern across days.

Test. On the next day, all animals received a single test session. This session consisted of $10 \mathrm{~min}$ of access to $1 \%$ almond in distilled water. Data from this session were used to confirm the effectiveness of the conditioning procedure and to allow intake-matched distribution of the animals for subsequent extinction and reconditioning treatments.

Extinction. Over the next 22 days, extinction was conducted in Groups Extinction, Retrain, and Unpaired. On each day they received $5 \mathrm{~min}$ of access to a $1 \%$ almond solution in distilled water. The 8 animals in Group No Extinction were spared extinction. On each day they received $5 \mathrm{~min}$ of access to distilled water.

Retraining. Over the next 18 days, the animals in Group Retrain were reexposed to the conditioning procedure. During this period they received conditioning treatments identical to those used in initial conditioning. All of the other animals received 5 min of exposure to distilled water during each day of this procedure.

Test. On each of the next 8 days, all animals received identical test sessions. Each session consisted of $5 \mathrm{~min}$ of exposure to $1 \%$ almond solution in distilled water.

\section{Results and Discussion}

The animals readily consumed the $20 \%$ polycose solution during the preexposure session. The mean intake across all animals was $15.6 \mathrm{ml}$.

There was less initial enthusiasm for the compounds containing the $1 \%$ almond on the first day of conditioning. The mean intake was $7.3 \mathrm{ml}$ for the animals in the three conditioning groups and $8.8 \mathrm{ml}$ for the unpaired group. However, over the course of conditioning, all animals increased their intake of this solution. On the final day of conditioning, the mean intake of almond was 17.5 for the paired groups and 17.0 for the unpaired group. The $2 \%$ polycose produced sufficient intake in the unpaired animals so that no difference was detectable as a function of pairing with the $20 \%$ polycose solution. However, in the test session, there was clear evidence of conditioning. The mean intake was $8.7 \mathrm{ml}$ for the paired groups and $3.5 \mathrm{ml}$ for the unpaired group [Mann-Whitney $U(24,8)=24.5$, $p<.01]$. Extinction by repeated exposure to almond in distilled water led to a decrease in intake to $3.4 \mathrm{ml}$ on the final day, which represented a reliable decrease in both Group Extinction and Group Retrain [Wilcoxon $T \mathrm{~s}(8) \leq 3$, $p$ s $<.05$ ]. Re-embedding of almond in $20 \%$ polycose produced rapid increase in intake to $17.3 \mathrm{ml}$ on the final retraining day.

Figure 1 shows the data of primary interest, from the test sessions. That figure shows clear evidence of conditioning in the greater intake by Group No Extinction than by Group Unpaired $[U(8,8)=0, p<.01]$. Similarly, the intake by Group Extinction was intermediate, marginally greater than in Group Unpaired $[U(8,8)=14, p<.06]$, 


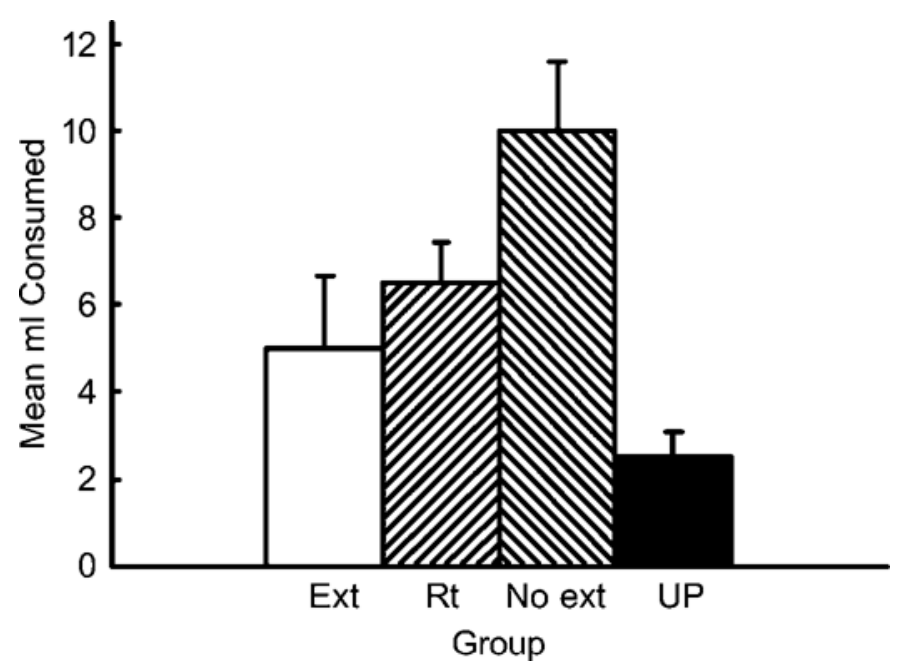

Figure 1. Mean intake $\cdot(+S E M)$ of the almond conditioned stimulus during the test sessions of Experiment 1. Groups No Extinction (No ext), Extinction (Ext), and Retrain (Rt) had all received simultaneous pairings of almond and polycose; Group Unpaired (UP) had received unpaired presentations. Groups Extinction and Retrain then received separate extinction presentations of almond. Group Retrain was retrained prior to the test.

but less than in Group No Extinction $[U(8,8)=9, p<.05]$. However, Group Retrain showed a level of performance more like that of Group Extinction than that of Group No Extinction. Group Retrain had an intake reliably less than that of Group No Extinction $[U(8,8)=12, p<.05]$, but not reliably greater than that of Group Extinction $[U(8,8)=17.5]$.

These results suggest that simultaneous pairing of almond and polycose led to learning that shows the properties of acquisition and extinction. However, there was relatively little indication that retraining after extinction produced reconditioning. In that regard, these results are similar to those reported for sensory preconditioning by Rescorla (1981).

\section{EXPERIMENT 2}

This experiment was a companion to Experiment 1, but with a sequential, rather than a simultaneous, relation between the almond and polycose. As in Experiment 1, three groups of rats received paired presentations of almond and polycose and one group received unpaired presentation. Of the three paired groups, one then received extinction and retraining, one received only extinction, and one received no further training, prior to a test. The question was whether sequential presentation of the same stimuli as used in Experiment 1 would produce the conventional observation of substantial retraining.

\section{Method}

Subjects and Apparatus

The subjects were 34 rats of the same strain and with histories similar to those in Experiment 1. The apparatus was that of Experiment 1.

\section{Procedure}

The procedures were the same as those of Experiment 1 with two exceptions. First, the paired presentations involved a 5 -min presentation of $1 \%$ almond mixed in $2 \%$ polycose followed immediately by $5-\min$ access to a $20 \%$ polycose solution. Second, extinction was carried out for 18 days, rather than 22 .

\section{Results and Discussion}

The intake of $20 \%$ polycose on the US preexposure day was similar to that in Experiment 1, $18.6 \mathrm{ml}$. Over the course of conditioning, the intake of the almond solution rose from 3.3 to 14.6 for the paired groups and from 2.5 to 16 in the unpaired group. As in Experiment 1, mixing almond in a $2 \%$ polycose solution yielded substantial intake in the unpaired group. The intakes of almond in distilled water on the single test day, however, revealed that conditioning had been successful. The mean intakes were 12.8 and 4.8 for the paired and unpaired groups, respectively $[U(26,8)=2.5, p<.01]$.

Over the course of extinction in Groups Extinction and Retrain, the mean intake of the almond in water solution dropped to $4.7 \mathrm{ml}$. With retraining, the intake in Group Retrain recovered to $14.3 \mathrm{ml}$ on the final day.

The data of most interest, for the 8 days of testing almond intake are shown in Figure 2. That figure displays a clear difference between the Unpaired control group and Group No Extinction $[U(8,9)=0, p<.01]$. This confirms the adequacy of the present procedures for producing conditioning. Group Extinction showed an intermediate level of intake, reliably greater than that for the Unpaired Group $[U(8,8)=11, p<.05]$, but reliably lower than that for Group No Extinction $[U(8,8)=5.5, p<.01]$. The 18 days of extinction had produced marked, but incomplete, re- 


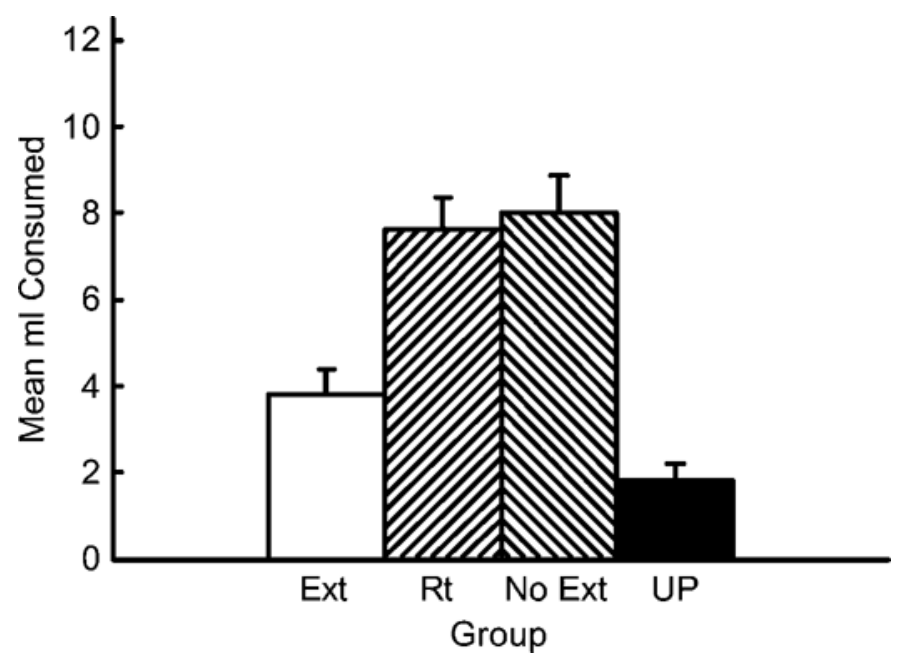

Figure 2. Mean intake $(+S E M)$ of the almond conditioned stimulus during the test sessions of Experiment 2. Groups No Extinction (No ext), Extinction (Ext), and Retrain (Rt) had all received sequential pairings of almond and polycose; Group Unpaired (UP) had received unpaired presentations. Groups Extinction and Retrain then received separate extinction presentations of almond. Group Retrain was retrained prior to the test.

duction in the evidence of initial conditioning. Finally, intake by Group Retrain was indistinguishable from that by Group No Extinction and reliably greater than that of either the unpaired or the extinguished group $[U(8,8) \mathrm{s}=1.5,9$, $p$ s $<.05]$.

These results confirm that, with sequential presentation, the present procedures are sufficient to generate good Pavlovian appetitive conditioning, extinction of that conditioning, and retraining following that extinction. Unlike simultaneous presentation, the sequential presentation of a flavor and $20 \%$ polycose apparently generates learned behavior that exhibits a relatively standard pattern of results.

\section{EXPERIMENT 3}

Experiments 1 and 2 suggest that sequential and simultaneous relations may yield different results in retraining. However, comparisons across experiments are always hazardous. Consequently, our intention in Experiment 3 was to carry out a direct comparison of the consequences of simultaneous and sequential presentation within the same experiment, and within the same animal. For this purpose, three groups of animals were given initial training with each of two flavors, almond and banana. One of the flavors was presented simultaneously, and one sequentially with a polycose US. Then both flavors were extinguished in two of the groups. For one of those extinction groups, both flavors were retrained with their original temporal relation to polycose. This design allows one to replicate within the same animal the sequential and simultaneous treatments of Experiments 1 and 2. It also allows one to compare the relative magnitudes of retraining with sequential and simultaneous conditioning.

\section{Method}

\section{Subjects and Apparatus}

The subjects were 36 rats of the same type and with histories similar to those of the rats in Experiments 1 and 2. The stimuli were the $20 \%$ and $2 \%$ polycose solutions used in Experiment 1, together with a $1 \%$ solution of almond or banana (McCormick imitation).

\section{Procedure}

Conditioning. As in Experiments 1 and 2, the animals received an initial day of exposure to the $20 \%$ polycose US. Over the course of the next 18 days, they received nine conditioning trials with each of the two flavors, presented for $5 \mathrm{~min}$. One of the flavors was presented in simultaneous compound with the $20 \%$ polycose. The other flavor was presented in compound with a $2 \%$ polycose solution and was followed immediately by $5 \mathrm{~min}$ of exposure to $20 \%$ polycose. For half of the animals, almond was given simultaneous conditioning and banana sequential conditioning; for the other half of the animals, the roles of almond and banana were interchanged. The flavors were presented in a double alternation pattern across days, beginning with sequential presentation on the first day.

Extinction. On each of the next 44 days, the 12 animals in Group Extinction and the 12 animals in Group Retrain received presentations of almond and banana in water. Flavors were presented one a day for $5 \mathrm{~min}$ in double alternation order over days. The 12 animals in Group No Extinction received unflavored water on these days.

Retraining. On each of the next 18 days, the animals in Group Retrain received reexposure to the original conditioning procedures. The animals in Groups Extinction and No Extinction received $10 \mathrm{~min}$ of water on each day.

Test. On each of the next 6 days, the animals received testing with almond and banana in water. Each day involved $10 \mathrm{~min}$ of either almond or banana given in a double alternation sequence across days. The data of most interest are the intakes of these solutions as a func- 
tion of whether they had been retrained or not and whether the original training had been with sequential or simultaneous presentation.

\section{Results and Discussion}

The consumption of the $20 \%$ polycose during initial exposure was somewhat less than that in the previous experiments, $11.4 \mathrm{ml}$.

Although the consumption of almond was slightly greater than that of banana at each stage of the experiment, none of the differences proved reliable. Moreover, the same data patterns were observed for both flavors. During training, extinction, and testing, the mean intakes were 14.2, 6.0, and 8.2 for almond; the comparable numbers were 13.7, 5.5, and 7.9 for banana. Over the course of conditioning, the level of intake rose from $8.8 \mathrm{ml}$ to $19.3 \mathrm{ml}$ for the simultaneously presented flavor and from 6.4 to 10.6 for the sequentially presented flavor. Because the flavor is presented in different concentrations of polycose in the case of simultaneous and sequential presentation, it is difficult to interpret the difference between them. Over the course of extinction, the intake fell from $8.0 \mathrm{ml}$ to $4.4 \mathrm{ml}$ for the simultaneously presented flavor and from 6.9 to 4.6 for the sequentially presented flavor. In both cases, the decrease in consumption was reliable $[T \mathrm{~s}(12) \leq 4, p \mathrm{~s}<.05]$. Retraining resulted in an increase in intake to 16.1 and $16.3 \mathrm{ml}$ on the final day, for the simultaneous and sequential stimulus, respectively.

Figure 3 shows the intake during the test period, when all subjects received $10 \mathrm{~min}$ of exposure to the flavors mixed with distilled water. The data patterns are highly similar to those from the comparable conditions of Experiments 1 and 2 .

\section{Sequential Conditioning}

To the right in the figure are shown the results for the flavor that had been trained in a sequential relation to the polycose. For that flavor, intake was less in Group Extinction than in Group No Extinction $[U(12,12)=28.5$, $p<.02]$. The intake for Group Retrain was substantially greater than that for Group Extinction $[U(12,12)=37$, $p<.05$ ], but not reliably different from that for Group No Extinction $[U(12,12)=60.5$, n.s.]. Consequently, with sequential training, there was evidence that extinction undermined intake and retraining reestablished it.

\section{Simultaneous Conditioning}

To the left of the figure are shown the results for the flavor that had been trained in a simultaneous relation to the polycose. For that flavor, intake was also less in Group Extinction than in Group No Extinction $[U(12,12)=13$, $p<.01]$. The intake for Group Retrain, however, was more like that for Group Extinction than that for Group No Extinction. The difference between intakes for Groups Retrain and No Extinction proved reliable $[U(12,12)=17.5$, $p<.01]$, whereas that between Group Retrain and Group Extinction did not $[U(12,12)=45.5$, n.s.]. With simultaneous conditioning, separate exposure to the flavor produced decreased intake, but retraining did not reestablish it.

\section{Simultaneous Versus Sequential Presentation}

Comparison of the intakes for the No Extinction Groups suggests that conditioning was more successful with simultaneous than with sequential presentation. During the test, there was greater intake of the simultaneously conditioned flavor [Wilcoxon $T(12)=5.5, p<.01$ ] Moreover, the magnitude of the difference in intake between the retrained and extinguished stimulus proved greater for the case of sequential conditioning than for simultaneous conditioning $[U(12,12)=36.5, p<.05]$. Similarly, the magnitude of the difference in intake between the retrained and not retrained stimulus proved greater for the case of simultaneous conditioning $[U(12,12)=13.5$, $p<.01]$. These comparisons confirm that there is an interaction between the degree to which retraining is successful and the use of sequential or simultaneous presentation mode.

\section{GENERAL DISCUSSION}

In these experiments, both simultaneous and sequential presentation of a flavor in compound with a positive consequence made that flavor more attractive. Moreover, in both cases, the separate presentation of the flavor without the consequence lead to extinction, as evidenced by a reduction in that attractiveness. However, reestablishing the flavor-consequence relation readily reestablished the attraction only in the case of sequential presentation. It appeared to have relatively little effect in the case of simultaneous presentation.

The difference in results with retraining seems unlikely to be attributable simply to the overall lower effectiveness of simultaneous conditioning. In Experiments 1 and 3, there was strong evidence of conditioning with a simultaneous presentation mode. Moreover, in Experiment 3 there was some evidence that in initial conditioning the simultaneous presentation mode actually produce a superior result.

The results for the simultaneous presentation mode agree with those of Freberg (1979) reported by Rescorla (1981) in the case of learning about two relatively neutral stimuli. Using flavors in sensory-preconditioninglike procedures, Freberg found excellent conditioning and extinction with simultaneous presentation, but little evidence of retraining. Apparently, Freberg's results are not confined to the use of relatively neutral stimuli but can also be obtained with simultaneous presentation of a CS and US.

One account of these results, suggested by Rescorla (1981), is that in some cases, the simultaneous presentation of two stimuli does not produce an association between them in the conventional sense. Rescorla argued that if A and B are presented simultaneously, the organism may not separately identify A and B as elements and then join them by an association. Instead, the animal may form a representation of a unitary AB compound. Subsequent separate presentation of either A or B may initially result in the animal's confusing them with the learned 


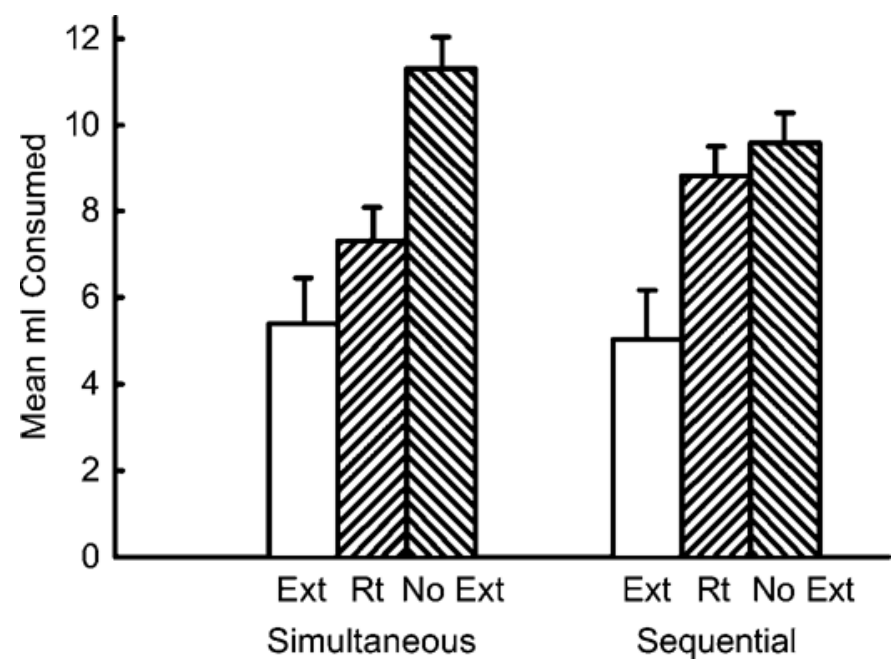

Figure 3. Mean intake $(+S E M)$ of the conditioned stimulus during test sessions of Experiment 3. The flavor CSs had been presented in either a simultaneous or a sequential relation with polycose. Group Extinction (Ext) had received extinction presentations and Group Retrain (Rt) had received both extinction and retraining. Group No Extinction (No Ext) received no separate presentations of the conditioned stimuli.

compound, and responding accordingly. However, with repeated separate presentations, an additional representation of the element may be formed, resulting in a discrimination between element and compound and reduced responding to the element. Consequently, what appears to be initial associative learning could in some cases be the formation of a single compound representation, whereas what looks like extinction could be the formation of a separate representation of a component. Under those circumstances, one might expect responding to a flavor to grow when it is presented jointly with an attractive substance, but then to decrease if the flavor is presented alone. However, separate presentation may not decrease responding because it removes the initial representation of the compound, but rather because it creates a separate representation of the element. Consequently, one would not expect that again presenting the flavor together with the substance would have the effect of reestablishing that compound representation. As a result, such retraining would not produce a recurrence of responding.

However, acknowledging that USs may have multiple features may provide alternative associative accounts of the results observed here. One possibility is that a US such as polycose has both sensory and nutritive features, each of which might enter into association with the CS (cf. Wagner \& Brandon, 1989). Moreover, simultaneous CS and US presentation might favor learning about the sensory features of the US, whereas sequential presentation might favor learning about the nutritive features. A variety of results suggest that with flavor CSs, learning about sensory features is especially strong with simultaneous presentation but more difficult with sequential presentation (e.g., Holman, 1975; Lavin, 1976; Rescorla,
1981). By contrast, learning about the postingestional, nutritive properties of the US can apparently occur even with substantial delays (e.g., Holman, 1975). Relatedly, Rescorla (1982) found evidence that simultaneous presentation of a tone and shock can increase the degree to which fear conditioning takes the form of learning about the sensory, as distinct from the response, features of the shock. If the conditioning based on sensory and other features of the US were to follow different associative rules, this could produce the pattern of results observed here. Unfortunately, there is little independent evidence to support that speculation.

An alternative associative interpretation of these data can be phrased in terms of the animal's ability to discriminate between the CS as presented during acquisition and the CS as presented during extinction. With standard Pavlovian conditioning, it is widely believed that extinction leaves intact the associations learned during acquisition but superimposes on them some new learning (see, e.g., Bouton, 1991). The level of performance observed following acquisition and extinction then depends on whether the animal identifies the current presentation of the CS as more similar to that present in acquisition or in extinction. One would expect that if a distinctive stimulus were present during acquisition and retraining, but absent during extinction and a subsequent test, then a tested CS might elicit a relatively low level of performance. One way to think about simultaneous CS and US presentations is that the US not only produces conditioning but also provides the CS with distinctive stimulus features that are absent at the time of testing. The sequentially presented CS, which was embedded in $2 \%$ polycose during acquisition and distilled water during extinction and test, might have 
a less substantial change in accompanying distinctive stimuli between training and extinction.

The role that this interpretation gives to the stimulus features of the US is like that attributed to contextual stimuli in Bouton's (1991) analysis of extinction. Like contexts present in extinction, the absence of those sensory US features comes to signal the nonreinforcement of the CS. Moreover, like such contexts, the initial absence of the stimulus features of the US might be expected to have relatively little immediate effect on initial performance in extinction. That is, one might expect there to be substantial evidence of conditioning during extinction even though the CS is presented alone without the concentrated polycose. According to this view, it is only over the course of extinction that the absence of the sensory features of polycose develops control over responding. However, as a result of that control, presenting the flavor again in compound with polycose during retraining would have little impact on subsequent responding when the flavor was tested alone, in the absence of those features.

It remains to be seen how general the retraining failure with simultaneous presentation will be. The present procedures, as well as those used by Freberg, have a number of unusual features, such as extended stimulus durations and self-administration of the CSs. Moreover, they have the property that conditioning is just as strong, if not stronger, with simultaneous, in comparison with sequential, stimulus presentation. But these results do suggest that not all instances of behavioral modification by joint stimulus presentation can be expected to show the same pattern of phenomena. They encourage the thought that multiple underlying mechanisms may be used by organisms to encode different temporal relations in their environment.

\section{REFERENCES}

Bouton, M. E. (1991). Context and retrieval in extinction and in other examples of interference in simple associative learning. In L. W. Dachowski \& C. F. Flaherty (Eds.), Current topics in animal learning: Brain, emotion, and cognition (pp. 25-53). Hillsdale, NJ: Erlbaum.

Capaldi, E. D, Hunter, M. J., \& LyN, S. A. (1997). Conditioning with taste as the CS in conditioned flavor preference learning. Animal Learning \& Behavior, 25, 427-436.

Domuan, M. (2003). The principles of learning and behavior (5th ed.). New York: Brooks/Cole.

FREBERG, L. A. (1979). Evidence supporting a perceptual learning model of the sensory preconditioning of flavors. Doctoral dissertation, UCLA.

Holman, E. W. (1975). Immediate and delayed reinforcers for flavor preferences in rats. Learning \& Motivation, 6, 91-100.

LAVIN, M. J. (1976). The establishment of flavor-flavor associations using a sensory preconditioning training procedure. Learning \& $\mathrm{Mo}$ tivation, 7, 173-183.

RESCORLA, R. A. (1981). Simultaneous associations. In P. Harzem \& M. Zeiler (Eds.), Advances in analysis of behavior (Vol. 2, pp. 4780). New York: Wiley.

RESCORLA, R. A. (1982). Simultaneous second-order conditioning produces S-S learning in conditioned suppression. Journal of Experimental Psychology: Animal Behavior Processes, 8, 23-32.

Rescorla, R. A., \& Durlach, P. J. (1981). Within-event learning in Pavlovian conditioning. In R. R. Miller \& N. E. Spear (Eds.), Information processing in animals: Memory mechanisms (pp. 81-111). Hillsdale, NJ: Erlbaum.

Rescorla, R. A., \& Freberg, L. (1978). The extinction of withincompound flavor associations. Learning \& Motivation, 9, 411-427.

Schwartz, B., Wasserman, E. A., \& Robbins, S. J. (2002). Psychology of learning and behavior (5th ed.). New York: W. W. Norton.

WAGNER, A. R., \& Brandon, S. E. (1989). Evolution of a structured connectionist model of Pavlovian conditioning (AESOP). In S. B. Klein \& R. R. Mowrer (Eds.), Contemporary theories: Pavlovian conditioning and the status of traditional learning theory (pp. 149189). Hillsdale, NJ: Erlbaum.

Westbrook, R. F., Duffield, T. Q., Good, A. J., Halligan, S., Seth, A. K., \& Swindbourne, A. L. (1995). Extinction of within-event learning is contextually controlled and subject to renewal. Quarterly Journal of Experimental Psychology, 48B, 357-375.

(Manuscript received October 7, 2003;

revision accepted for publication January 22, 2004.) 